\title{
DØ CRYO TO DØ PHYSICS DAQ COMMUNICATIONS LINK
}

\author{
ENGINEERING NOTE \\ 3740.515-EN-420
}

Dan Markley

7 MARCH 95 
OVERVIEW: This engineering note documents a communication link between the $D \emptyset$ cryo/gas control system and the $D \emptyset$ physics data aquisition computer system. This note is being written well after the work has been completed, in order to document this communications link, and to satisfy needs of planned upgrades. These upgrades are the DØ Super Conducting Solenoid, VLPC system, and Silicon Detector System.

The $\mathrm{D} \emptyset$ cryo/gas control system is a Programmable Logic Controller based process control system. It is responsible for controlling many of the physical parameters of the D $\varnothing$ experiment, such as Calorimeter cooling, vacuum, Instrument air, TRD gas pressures and flows, WAMUS pressures and flows, SAMUS pressure and flows, etc. It works independent of the Physics DAQ system.

There is a need for the Physics DAQ system to record some of these process values with the $D \emptyset$ physics data. This process data will later be used to calibrate certain features of the Physics data during reconstruction. The process data is also used to interlock (via the Physics DAQ system) some of the other DØ systems such as the TRD high voltage system. Some of the Process data values will be continuously monitored and will stop the Physics data taking of the experiment if it is out of tolerance.

Components: The PLC uses a Basic programming language I/O Module \#505-7101 with 2 serial ports. 1 of the ports communicates with Token ring node \#070D located in room \#510. The other Basic module serial port is used to control and program the module from an IBM compatible computer. Both ports are using an RS232 serial interface.

The Token ring node \#070D has a serial server program running that was programmed by Bob Goodwin and is maintained by Greg Cisco. This program is automatically loaded when there is a power recovery after an upset.

How It Works: The PLC is constantly updating both input and output at a rate of about $40 \mathrm{~ms} / \mathrm{IO}$ update cycle. The Basic Module will download the listed $\mathrm{I} / \mathrm{O}$ into it's memory then transmit these values over the serial link to the Token Ring Node at a rate of about 1 complete cycle/3 minutes. This data is transmitted in the form of something known as an $\mathrm{S} 1$ record. These records are continually updated over and over to a block of memory in the Token Ring Node. 
The Physics DAQ computers will update it's database from this block of memory in the Token Ring Node. There is a time stamp sent along with this block of data. The Physics DAQ section checks to see that the time stamp is updated every so often, and if it's not, will cause an alarm in the $D \varnothing$ main control room.

The current name of the Basic module executable program is S-REC-PR. The software that :runs on the compatible computer that programs the Basic module is called B-TERM. It can and does run under Windows for Workgroups and Windows NT. B-TERM can but doesn't need to run once the Basic module is programmed and in the run mode. It can be run however to check the output of either serial port. B-TERM itself, is not much more than a terminal emultor.

Players: This project was completed in September of 1993.

Dan Markley was the organizer of the project.

Mike Mcgee was the principal Basic programmer of the Basic Module.

Greg Cisko was a basic programming consultant and the Token Ring Node coordinator.

Bob Angstadt was a basic programming consultant.

Bob Goodwin was the Token Ring Node software writer. Laura Paterno was the DAQ system programmer.

Expansion: There are 150 memory locations continuously transmitted to the Token ring node. Each memory location stores a 12 bit word. Of those 150 locations only about 100 contain assigned data. 50 locations will be abandoned after run $1 \mathrm{~B}$ due to the removal of the TRD system. This will leave about 100 memory locations for future transmittal of process data to the Physics DAQ systems. This should be more than enough to cover the planned Solenoid, VLPC, Silicon detector etc.

What this means, is that in order to expand the number of communicated $\mathrm{I} / \mathrm{O}$, none of the internal programming of the com link software will have to be altered.

\section{Also Included in This Note:}

1. A hard copy of the Basic code currently running in the Basic I/O module.

2. A spread sheet that defines the data that is transferred from the PLC to the Token Ring Node. 


\begin{tabular}{|c|c|c|c|c|c|c|c|c|c|}
\hline$\#$ & BASE & SLOT/PT & VME CHANNEL & V ADD & ADDRESS & TAG & DESCFPTION & SEX & RANGE \\
\hline 1 & & & 300 & V3701 & & & YEAR & & \\
\hline 2 & & & 301 & V3702 & & & MONTH & & \\
\hline 3 & & & 302 & V3703 & & & DAY & & \\
\hline 4 & & & 303 & V3704 & & & HOUR & & \\
\hline 5 & & & 304 & V3705 & & & MNUTE & & \\
\hline 6 & & & 305 & V3706 & & & SECONDS & & \\
\hline 7 & & & 306 & V3707 & & & & & \\
\hline 8 & & & 307 & V3708 & & & & & \\
\hline 9 & & & 308 & V3709 & & & & & \\
\hline 10 & & & 309 & V3710 & & & & & \\
\hline 11 & 1 & $1 / 1$ & $30 \mathrm{~A}$ & V3711 & $W \times 2049$ & DET 1P & DET 1 TO ATM DIFFERENTIAL & $(4-20 \mathrm{ma})$ & $-5^{\prime \prime} \mathrm{TO}+5^{\prime \prime} \mathrm{H} 2 \mathrm{O}$ \\
\hline 12 & 1 & $1 / 2$ & $30 \mathrm{~B}$ & V3712 & 2050 & GAP 1P & GAP 1 TO DET 1 DIFFERENTIAL & $(4-20 \mathrm{ma})$ & $-5^{\prime \prime} \mathrm{TO}^{\circ} 5^{\prime \prime} \mathrm{H} 2 \mathrm{O}$ \\
\hline 13 & 1 & $1 / 3$ & $30 \mathrm{C}$ & V3713 & 2051 & RAD IP & RAD 1 TO GAP 1 DIFFERENTIAL & $(4-20 \mathrm{ma})$ & $-5^{\prime \prime} \mathrm{TO}+5^{n} \mathrm{H} 2 \mathrm{O}$ \\
\hline 14 & 1 & $1 / 4$ & 300 & V3714 & 2052 & & & & \\
\hline 15 & 1 & $1 / 5$ & $30 E$ & V3715 & 2053 & DET 2P & DET 2 TO ATM DIFFERENTIAL & $(4-20, \mathrm{ma})$ & $-5^{\prime \prime} \mathrm{TO}+5^{\mathrm{N}} \mathrm{H} 2 \mathrm{O}$ \\
\hline 16 & 1 & $1 / 6$ & $30 \mathrm{~F}$ & V3716 & 2054 & GAP $2 P$ & GAP 2 TO DET 2 DIFFERENTIAL & $(4-20 \mathrm{ma})$ & $-5^{n} \mathrm{TO}+5^{n} \mathrm{H} 2 \mathrm{O}$ \\
\hline 17 & 1 & $1 / 7$ & 310 & V3717 & 2055 & RAD 2P & RAD 2 TO GAP 2 DIFFERENTIAL & (4-20 ma) & $-5^{n} \mathrm{TO}+5^{n} \mathrm{H} 2 \mathrm{O}$ \\
\hline 18 & 1 & $1 / 8$ & 311 & V3718 & 2056 & & & $=$ & \\
\hline 19 & 1 & $2 / 1$ & 312 & V3719 & WX2057 & DET 3P & DET 3 TO ATM DIFFERENTIAL & $(4-20 \mathrm{ma})$ & $-5^{\prime \prime} \mathrm{TO}^{\circ} 5^{\mathrm{n}} \mathrm{H} 2 \mathrm{O}$ \\
\hline 20 & 1 & $2 / 2$ & 313 & V3720 & 2058 & GAP 3P & GAP 3 TO DET 3 DIFFERENTIAL & $(4-20 \mathrm{ma})$ & $-5^{\prime \prime} \mathrm{TO}+5^{\prime \prime} \mathrm{H} 2 \mathrm{O}$ \\
\hline 21 & 1 & $2 / 3$ & 314 & V3721 & 2059 & RAD 3P & RAD 3 TO GAP 3 DIFFERENTIAL & $(4-20 \mathrm{ma})$ & $-5^{\prime \prime} \mathrm{TO}+5^{n \prime} \mathrm{H} 2 \mathrm{O}$ \\
\hline 22 & 1 & $2 / 4$ & 315 & V3722 & 2060 & & & & \\
\hline 23 & 1 & $2 / 5$ & 316 & v3723 & 2061 & & & & \\
\hline 24 & 1 & $2 / 6$ & 317 & V3724 & 2062 & & & & \\
\hline 25 & 1 & $2 / 7$ & 318 & V3725 & 2063 & & & & \\
\hline 26 & 1 & $2 / 8$ & 319 & V3726 & 2064 & & & & \\
\hline 27 & 1 & $3 / 1$ & $31 \mathrm{~A}$ & V3727 & $W \times 2065$ & RAD 1FL OW & RAD 1 FLOW & (0-5 VOLTS) & $0-70 \mathrm{LPH}$ \\
\hline 28 & 1 & $3 / 2$ & $31 \mathrm{~B}$ & V3728 & 2066 & GAP 1FL OW & GAP 1 FLOW & (0.5 VOLTS) & $0-15 \mathrm{LPH}$ \\
\hline 29 & 1 & $3 / 3$ & $31 \mathrm{C}$ & V3729 & 2067 & DET 1FL OW & DET 1 FLOW & (0.5 VOLTS) & $0.40 \mathrm{LPH}$ \\
\hline 30 & 1 & $3 / 4$ & 31D & V3730 & 2068 & RAD 2FL OW & \begin{tabular}{|r|} 
RAD 2 FLOW \\
\end{tabular} & (0-5 VOLTS) & $0-100 \mathrm{LPH}$ \\
\hline 31 & 1 & $3 / 5$ & 31E & V3731 & 2069 & GAP 2FL OW & GAP 2 FLOW & (0.5 VOLTS) & $0.25 \mathrm{LPH}$ \\
\hline 32 & 1 & $3 / 6$ & $31 F$ & V3732 & 2070 & DET 2FL OW & DET 2 FLOW & (0.5 VOLTS) & $0-50 \mathrm{LPH}$ \\
\hline
\end{tabular}




\begin{tabular}{|c|c|c|c|c|c|c|c|c|c|}
\hline$\#$ & BASE & SLOT/PT & VME CHANNEL & V ADD & ADDRESS & TAG & DESCPIPTION & SEX & RANGE \\
\hline 33 & 1 & $3 / 7$ & 320 & V3733 & 2071 & RAD $3 F L$ OW & RAD 3 FLOW & (0.5 VOLTS) & $0-120 \mathrm{LPH}$ \\
\hline 34 & 1 & $3 / 8$ & 321 & V3734 & 2072 & GAP 3FL OW & GAP 3 FLOW & (0-5 VOLTS) & $0-25 \mathrm{LPH}$ \\
\hline 35 & 1 & $4 / 1$ & 322 & V3735 & $W \times 2073$ & DET 3FL OW & DET 3 FLOW & (0-5 VOLTS) & $0-65 \mathrm{LPH}$ \\
\hline 36 & 1 & $4 / 2$ & 323 & V3736 & 2074 & & & & \\
\hline 37 & 1 & $4 / 3$ & 324 & V3737 & 2075 & & & & \\
\hline 38 & 1 & $4 / 4$ & 325 & V3738 & 2076 & & & & \\
\hline 39 & 1 & $4 / 5$ & 326 & V3739 & 2077 & & & & \\
\hline 40 & 1 & $4 / 6$ & 327 & V3740 & 2078 & & & & \\
\hline 41 & 1 & $4 / 7$ & 328 & V3741 & 2079 & & & & \\
\hline 42 & 1 & $4 / 8$ & 329 & V3742 & 2080 & PLA TAT M & PLATFORM ATM PRESS. TRANSMITTER & $0-5$ VOLTS $=800$ & $1060 \mathrm{MBAR}$ \\
\hline 43 & 2 & $1 / 1$ & 32A & V3743 & $W \times 2209$ & TFD LPS & L.P.S. & $(4-20 \mathrm{MA})$ & $0-30$ PSIG \\
\hline 44 & 2 & $1 / 2$ & 32B & V3744 & 2210 & TRD SUC & SUCTIONPRESSURE & $(4-20 \mathrm{MA})$ & -5 TO 5 " $\mathrm{H} 20$ \\
\hline 45 & 2 & $1 / 3$ & $32 \mathrm{C}$ & V3745 & 2211 & TFD CDI S & TRDPUMP DISCHARGE PRESSURE & $(4-20 \mathrm{MA})$ & $0.30 \mathrm{PSIG}$ \\
\hline 46 & 2 & $1 / 4$ & 32D & V3746 & 2212 & TRD VOL 0 & VOLøPRESSURE & $(4-20 \mathrm{MA})$ & $0-30$ PSIG \\
\hline 47 & 2 & $1 / 5$ & 32E & V 3747 & 2213 & TRD XP LT & XENONPRESSURE TO PLATFORM & $(4-20, M A)$ & $0-30$ PSIG \\
\hline 48 & 2 & $1 / 6$ & $32 F$ & V3748 & 2214 & TRD CAN & CANARYPRESSURE & $(4-20 \mathrm{MA})$ & -5 TO $5 \mathrm{n} \mathrm{H} 2 \mathrm{O}$ \\
\hline 49 & 2 & $1 / 7$ & 330 & V3749 & 2215 & & TRD VAC PUMP VACUUM PIRANNI & $(4-20 \mathrm{MA})$ & $0-1000$ TORR \\
\hline 50 & 2 & $1 / 8$ & 331 & $\vee 3750$ & 2216 & TPO H2O & TRD MOISTURE ANALYZER & $(4-20=\mathrm{MA})$ & 100-10K PPM \\
\hline 51 & 2 & $2 / 1$ & 332 & V3751 & $W \times 2217$ & TRD TXE RL & XENON TOTAL FLOW METER & $0-5$ VDC & $0-150 \mathrm{LPH}$ \\
\hline 52 & 2 & $2 / 2$ & 333 & V3752 & 2218 & ROO MAT M & TRD ROOMATMPRESSURE & $0-5 \mathrm{VOLTS}=800$ & 1060 MBAR \\
\hline 53 & 2 & $2 / 3$ & 334 & V3753 & 2219 & & & & \\
\hline 54 & 2 & $2 / 4$ & 335 & V3754 & 2220 & & & & \\
\hline 55 & 2 & $2 / 5$ & 336 & V3755 & 2221 & & & & \\
\hline 56 & 2 & $2 / 6$ & 337 & V3756 & 2222 & & & & \\
\hline 57 & 2 & $2 \pi$ & 338 & V3757 & V2223 & & & & \\
\hline 58 & 2 & $2 / 8$ & 339 & V3758 & 2224 & TRD 02 & \begin{tabular}{|r} 
O2 ANAL YZER \\
\end{tabular} & $(4-20 \mathrm{MA})$ & $0-10$ PPM \\
\hline 59 & 1 & $6 / 1$ & 33A & $\vee 3759$ & $W \times 2089$ & TRD PLT TPM & TRD PLATFORM GAS TEMPERATURE & $(-200$ TO 850 & CELCIUS \\
\hline 60 & 3 & $1 / 3$ & 33B & V3760 & 515 & TRD FM TMP & TRD ROOM GAS TEMPERATURE & $(-75 \mathrm{TO}+175$ & CELCIUS \\
\hline 61 & 0 & $1 / 1$ & $33 \mathrm{C}$ & V3761 & $w \times 0001$ & PT $233 \quad \mathrm{~A}$ & \begin{tabular}{|c|} 
CCUPPER VPT \\
\end{tabular} & $(4-20 \mathrm{MA})$ & 14-27 PSIA \\
\hline 62 & 0 & $1 / 2$ & 33D & V3762 & 2 & PT 234 & CCLOWER VPT & $(4-20 \mathrm{MA})$ & 14-27 PSIA \\
\hline 63 & 0 & $1 / 3$ & 33E & V3763 & 3 & PT 240 & CC MIDDLE VPT & $(4-20 \mathrm{MA})$ & 14-27 PSIA \\
\hline 64 & 0 & $1 / 4$ & 33F & V3764 & 4 & DPT 235 & CC TOP TO BOTTOM VPT DIFFERENTIAL & $(4-20 \mathrm{MA})$ & 0.2 .5 PSIA \\
\hline
\end{tabular}

Page 2 


\begin{tabular}{|c|c|c|c|c|c|c|c|c|c|c|}
\hline \# & BASE & SLOT/PT & VME CHANNEL & V ADD & ADDRESS & & TAG & DESCRIPTION & SEX & RANGE \\
\hline 65 & 0 & $1 / 5$ & 340 & V3765 & 5 & PT 2 & 210 & CC CONDENSOR OUTLET PRESSURE & $(4-20 \mathrm{MA})$ & 0.75 PSIA \\
\hline 66 & 0 & $1 / 6$ & 341 & V3766 & 6 & PT 2 & 204 & CC CRYOSTAT PRESSURE MONITOR & $(4-20 \mathrm{MA})$ & 14-27 PSIA \\
\hline 67 & 0 & $1 / 7$ & 342 & V3767 & 7 & PT & 230 & CC CRYOSTAT PRESSURE CONTROL & (4-20 MA) & 14-27 PSIA \\
\hline 68 & 0 & $1 / 8$ & 343 & V3768 & 8 & DPT 2 & 222 & CC VPT DIFFERENTIAL FROM TOP TO BO & $(4-20 \mathrm{MA})$ & $0-10 \mathrm{PSI}$ \\
\hline 69 & 0 & $2 / 1$ & 344 & V3769 & $W \times 0009$ & & & & & \\
\hline 70 & 0 & $2 / 2$ & 345 & V3770 & 10 & 且 & 223 & CC ARGON LKUID OPERATING LEVEL & $(4-20 \mathrm{MA})$ & 0 TO $100 \%$ \\
\hline 71 & 0 & $2 / 8$ & 346 & V3771 & 16 & PT & 333 & $\begin{array}{l}\text { SEC UPPER VPT } \\
\end{array}$ & (4-20 MA) & 14-27 PSIA \\
\hline 72 & 0 & $3 / 1$ & 347 & V3772 & $W \times 0017$ & PT & 334 & SEC LOWER VPT & $(4-20 \mathrm{MA})$ & 14-27 PSIA \\
\hline 73 & 0 & $3 / 2$ & 348 & V3773 & 18 & PT & 340 & SEC MIDDLE VPT & $(4-20 \mathrm{MA})$ & 14-27 PSIA \\
\hline 74 & 0 & $3 / 5$ & 349 & V3774 & 21 & PT & 304 & SEC CRYOSTAT PRESSURE MONTTOR & $(4-20 \mathrm{MA})$ & 14-27 PSIA \\
\hline 75 & 0 & $4 / 5$ & $34 \mathrm{~A}$ & V3775 & 29 & PT & 330 & SEC CRYOSTAT PRESSURE CONTROL & $(4-20 \mathrm{MA})$ & 14-27 PSIA \\
\hline 76 & 0 & $4 / 6$ & 34B & V3776 & 30 & DPT 3 & 322 & SEC DIFFERENTIAL TOP TO BOT(level) & $(4-20 \mathrm{MA})$ & $0-10 \mathrm{PSI}$ \\
\hline 77 & 0 & $5 / 3$ & $34 \mathrm{C}$ & V3777 & 35 & 日 3 & 323 & SEC ARGONLIQUID OPERATING LEVEL & $(4-20 \mathrm{MA})$ & 0 TO $100 \%$ \\
\hline 78 & 0 & $3 / 4$ & 34D & V3778 & $w \times 20$ & PT 3 & 310 & SEC CONDENSOROUTLET PRESSURA & $(4-20 \mathrm{MA})$ & $0-75$ PSIA \\
\hline 79 & 0 & $7 / 2$ & 34E & V3779 & 50 & PT 1 & 133 & \begin{tabular}{|c|} 
NECUPPER VPT \\
\end{tabular} & $(4-20, M A)$ & 14-27 PSIA \\
\hline 80 & 0 & $7 / 3$ & 34F & V3780 & 51 & PT & 134 & NEC LOWER VPT & $(4-20 \mathrm{MA})$ & 14-27 PSIA \\
\hline 81 & 0 & $7 / 4$ & 350 & V3781 & 52 & PT & 140 & $\begin{array}{l}\text { NEC MIDDLE VPT } \\
\end{array}$ & $(4-20 \mathrm{MA})$ & 14-27 PSIA \\
\hline 82 & 0 & $7 / 6$ & 351 & V3782 & 54 & PT 1 & 110 & NECCONDENSOROUTLET PRESSURE & $(4-20=\mathrm{MA})$ & $0-75$ PSIA \\
\hline 83 & 0 & $7 / 7$ & 352 & V3783 & 55 & PT 1 & 104 & NEC CRYOSTAT PRESSURE MONITOR & $(4-20 \mathrm{MA})$ & 14-27 PSIA \\
\hline 84 & 0 & $7 / 8$ & 353 & V3784 & 56 & PT & 130 & NEC CRYOSTAT PRESSURE CONTROL & (4-20 MA) & 14-27 PSIA \\
\hline 85 & 0 & $8 / 1$ & 354 & V3785 & $W \times 0057$ & DPT 1 & 122 & NEC DIFFERENTIAL TOP TO BOT(level) & $(4-20 \mathrm{MA})$ & $0-10 \mathrm{PSI}$ \\
\hline 86 & 0 & $8 / 2$ & 355 & V3786 & 58 & & & & & \\
\hline 87 & 0 & $8 / 3$ & 356 & V3787 & 59 & 日 1 & $123 \mathrm{~A}$ & NEC ARGON LIQUID OPERATING LEVEL & $(4-20 \mathrm{MA})$ & 0 TO $100 \%$ \\
\hline 88 & 3 & $3 / 1$ & 357 & V3788 & WX529 & FTR & $1 \mathrm{FEC}$ & WAMUS FLOW TO VENT & $0-5$ VDC & $0-100$ SLPM \\
\hline 89 & 3 & $3 / 2$ & 358 & V3789 & 530 & $\mathrm{FT}$ & AR & WAMUS ONE PASS FLOW & $0-5$ VDC & 0-100 SLPM \\
\hline 90 & 3 & $3 / 3$ & 359 & V3790 & 531 & $\mathrm{FT}$ & $1 \mathrm{PEC}$ & WAMUS FLOWTO CHAMBERS & 0.5 VDC & D-106.16 SLPM \\
\hline 91 & 3 & $3 / 4$ & 35A & V3791 & 532 & PT & $2 \mathrm{PEC}$ & WAMUS PUMP DISCHARGE PRESS & $(4-20 \mathrm{MA})$ & $0-60 \mathrm{PSI}$ \\
\hline 92 & 3 & $3 / 5$ & 35B & V3792 & 533 & PT & 3 PEC & WAMUS CHAMBER INLET PRESS & $(4-20 \mathrm{MA})$ & $0-25 " \mathrm{H} 2 \mathrm{O}$ \\
\hline 93 & 3 & $4 / 1$ & $35 \mathrm{C}$ & V3793 & WX537 & SAM & $\mathrm{FL}$ & SAMUS CHAMBER SUPPLY FLOW & $0-5$ VDC & $0-5.0$ SLPM \\
\hline 94 & 3 & $4 / 2$ & 35D & V3794 & 538 & CO & 02 & WAMUS CO2 (ANARAD ANALYZER) & 1-5 VDC & $0-10 \%$ \\
\hline 95 & 3 & $4 / 3$ & 35E & V3795 & 539 & PE 0 & & WAMUS FREON (ROSEMOUNT ANAL & 4-20MA & $0-20 \%$ \\
\hline 96 & 3 & $4 / 4$ & 35F & V3796 & 540 & $\mathrm{H} 2$ & 20 & WAMUS H2O (PANAMETRICS ANAL & $0-2$ VDC & $0-250$ PPM \\
\hline
\end{tabular}




\begin{tabular}{|c|c|c|c|c|c|c|c|c|c|}
\hline$\#$ & BASE & SLOT/PT & VME CHANNEL & V ADD & ADDRESS & TAG & DESCRIPTION & $\operatorname{sex}$ & RANGE \\
\hline 97 & 3 & $4 / 5$ & 360 & V3797 & 541 & $\mathrm{O} 2$ & WAMUS O2 (CUSTOM ANALYZER) & 4-20MA & O-200 PPM \\
\hline 98 & 3 & $4 / 6$ & 361 & V3798 & 542 & SAM PT9 4 & SAMUS MIXING TANK PRESSURE & 4-20 MA & $0.50 \mathrm{PSI}$ \\
\hline 99 & 3 & $4 / 7$ & 362 & V3799 & 543 & SAM SUP & SAMUS PRESSURE SUPPLYTOCHAM & $4-20 \mathrm{MA}$ & $0-138.4^{\prime \prime} \mathrm{H} 2 \mathrm{O}$ \\
\hline 100 & & & 363 & V3800 & & & & & \\
\hline 101 & & & 364 & V3801 & & & & & \\
\hline 102 & & & 365 & V3802 & & & & & \\
\hline 103 & & & 366 & V3803 & & & & & \\
\hline 104 & & & 367 & V3804 & & & & & \\
\hline 105 & & & 368 & V3805 & & & & & \\
\hline 106 & & & 369 & V3806 & & & & & \\
\hline 107 & & & $36 \mathrm{~A}$ & V3807 & & & & & \\
\hline 108 & & & $36 \mathrm{~B}$ & V3808 & & & & & \\
\hline 109 & & & $36 \mathrm{C}$ & V3809 & & & & & \\
\hline 110 & & & 36D & V3810 & & & & & \\
\hline 111 & & & $36 E$ & V3811 & & & & $\therefore$ & \\
\hline 112 & & & $36 \mathrm{~F}$ & V3812 & & & & & \\
\hline 113 & & & 370 & V3813 & & & & & \\
\hline 114 & & & 371 & V3814 & & & & $=$ & \\
\hline 115 & & & 372 & V3815 & & & & & \\
\hline 116 & & & 373 & V3816 & & & & & \\
\hline 117 & & & 374 & V3817 & & & & & \\
\hline 118 & & & 375 & V3818 & & & & & \\
\hline 119 & & & 376 & V3819 & & & & & \\
\hline 120 & & & 377 & V3820 & & & & & \\
\hline 121 & & & 378 & V3821 & & & & & \\
\hline 122 & & & 379 & V3822 & & & & & \\
\hline 123 & & & $37 \mathrm{~A}$ & V3823 & & & & & \\
\hline 124 & & & $37 \mathrm{~B}$ & V3824 & & & & & \\
\hline 125 & & & $37 \mathrm{C}$ & V3825 & & & & & \\
\hline 126 & & & 370 & V3826 & & & & & \\
\hline 127 & & & 37E & V3827 & & & & & \\
\hline 128 & & & $37 F$ & V3828 & & & & & \\
\hline
\end{tabular}




\begin{tabular}{|c|c|c|c|c|c|c|c|c|c|}
\hline 4 & BASE & SLOT/PT & VME CHANNEL & V ADD & ADDRESS & TAG & DESCRIPTION & SEX & RANGE \\
\hline 129 & & & 380 & V3829 & & & & & \\
\hline 130 & & & 381 & V3830 & & & & & \\
\hline 131 & & & 382 & V3831 & & & & & \\
\hline 132 & & & 383 & V3832 & & & & & \\
\hline 133 & & & 384 & V3833 & & & & & \\
\hline 134 & & & 385 & $V 3834$ & & & & & \\
\hline 135 & & & 386 & V3835 & & & & & \\
\hline 136 & & & 387 & V3836 & & & & & \\
\hline 137 & & & 388 & V3837 & & & & & \\
\hline 138 & & & 389 & $V 3838$ & & & & & \\
\hline 139 & & & $38 \mathrm{~A}$ & V3839 & & & & & \\
\hline 140 & & & $38 \mathrm{~B}$ & V3840 & & & & & \\
\hline 141 & & & $38 \mathrm{C}$ & V3841 & & & & & \\
\hline 142 & & & 38D & V3842 & & & & & \\
\hline 143 & & & $38 E$ & V3843 & & & & $\therefore$ & \\
\hline 144 & & & $38 \mathrm{~F}$ & V3844 & & & & & \\
\hline 145 & & & 390 & V3845 & & & & & \\
\hline 146 & & & 391 & V3846 & & & & 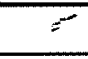 & \\
\hline 147 & & & 392 & V3847 & & & & & \\
\hline 148 & & & 393 & $\mathrm{~V} 3848$ & & & & & \\
\hline 149 & & & 394 & V3849 & & & & & \\
\hline 150 & & & 395 & V3850 & & & & & \\
\hline
\end{tabular}


S-REC-PR

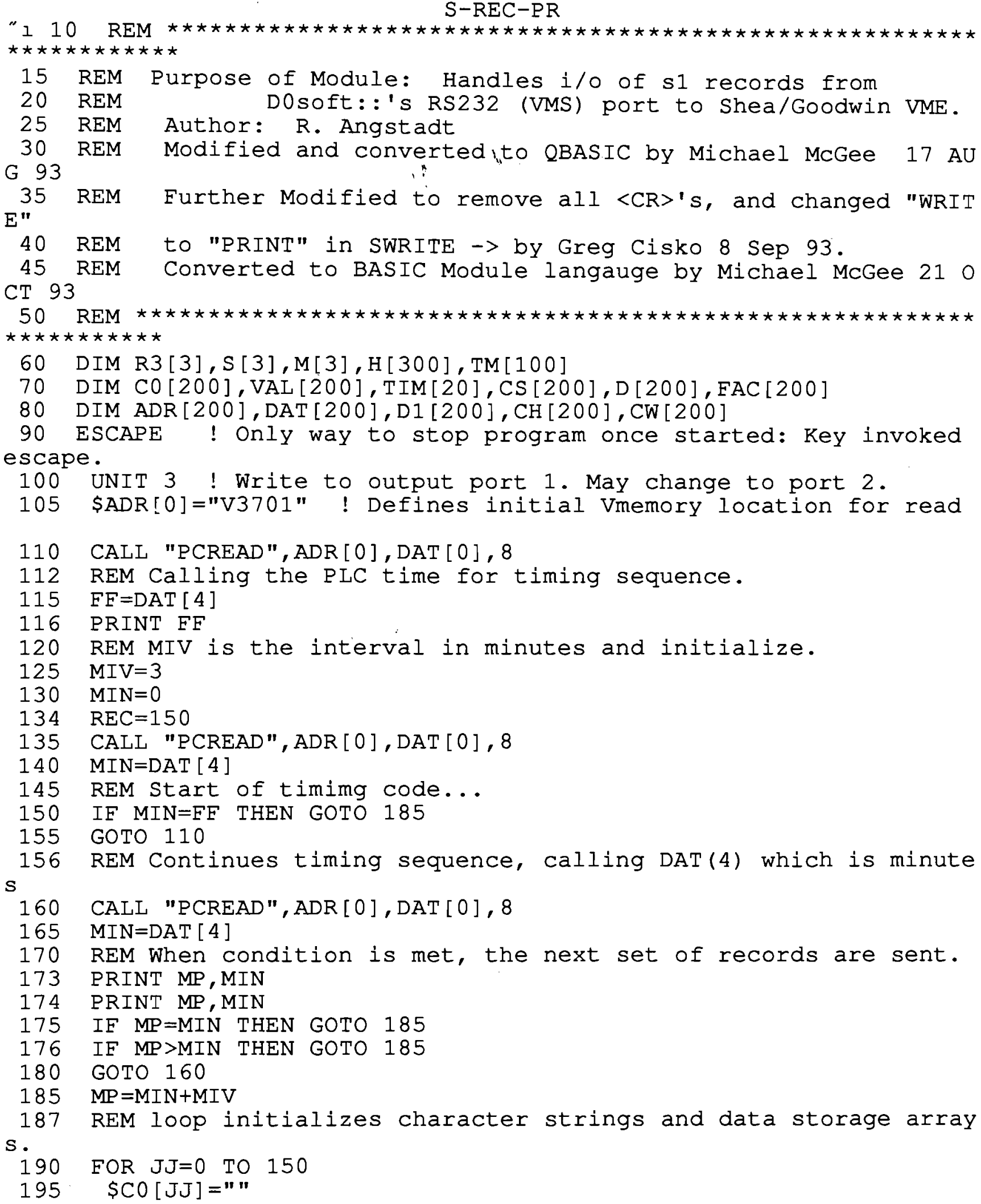




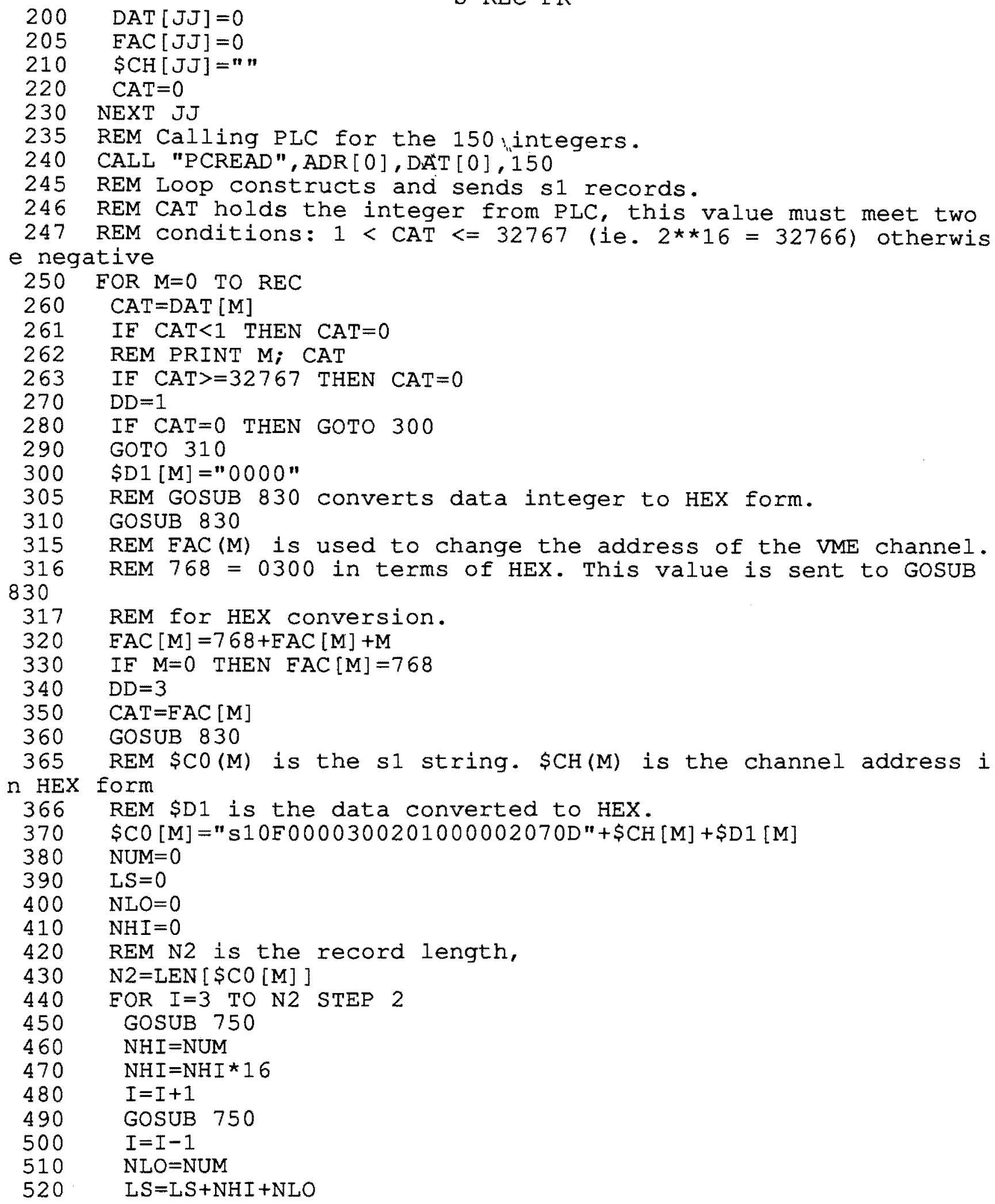




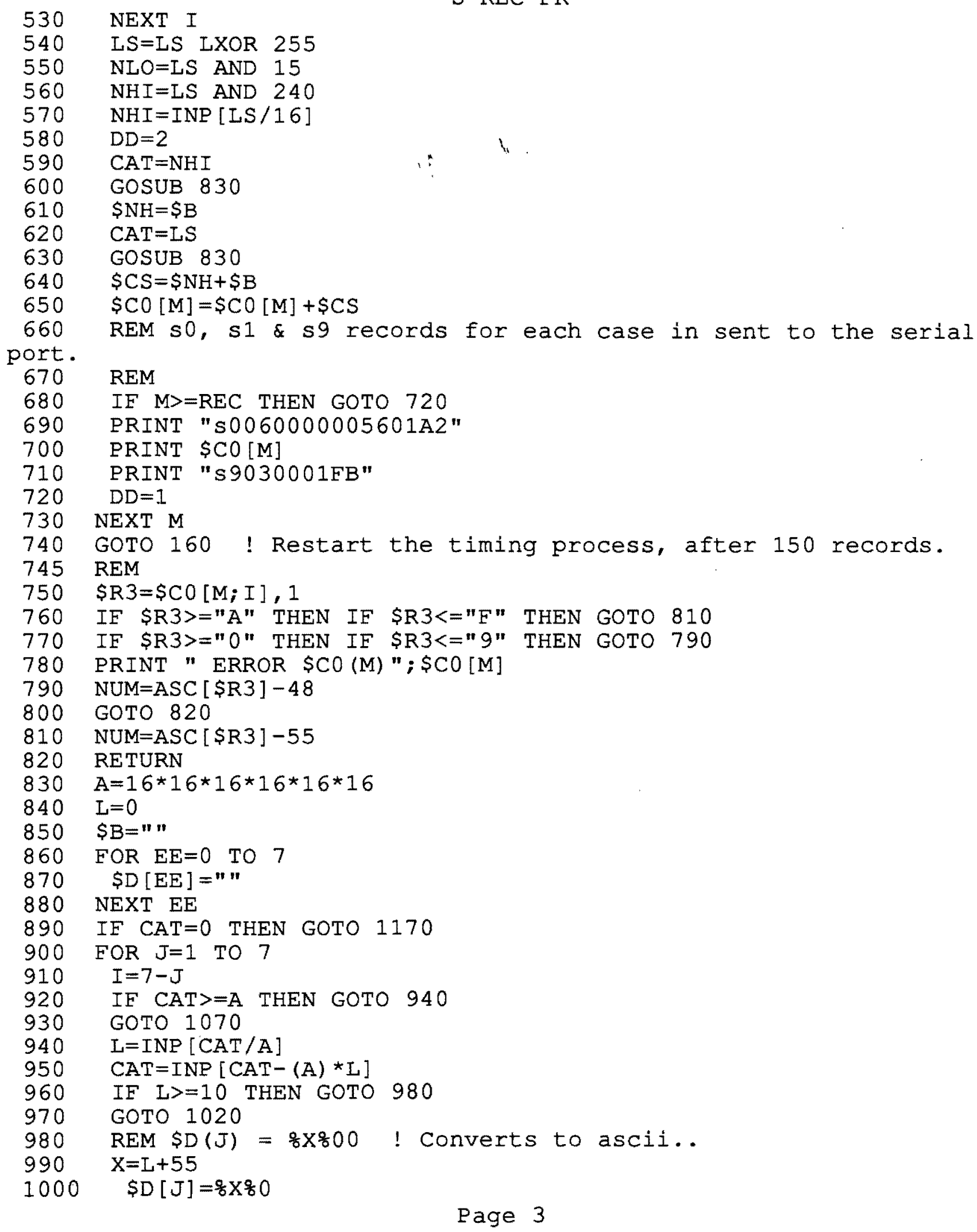




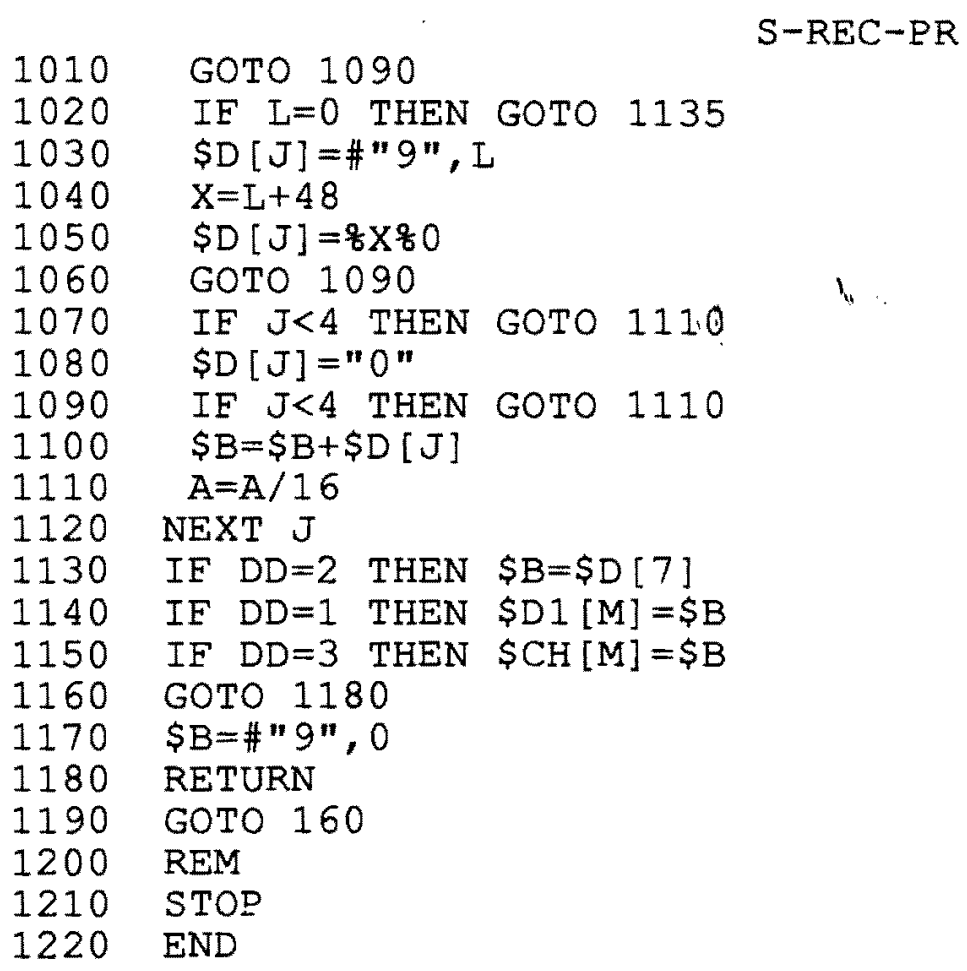

